\title{
Influência de Políticas de Ação Afirmativa no Perfil Sociodemográfico de Estudantes de Medicina de Universidade Brasileira
}

\section{Influence of Affirmative Action Policies on Socio Demographic Profile of Medical Students from a Brazilian University}

\author{
Maria Laura Alves de Melo Silval \\ Eliana Amaral ${ }^{I}$ \\ Helymar da Costa Machado ${ }^{I}$ \\ Silvia Maria Riceto Ronchim Passeri ${ }^{I}$ \\ Joana Fróes Bragança ${ }^{I}$
}

\section{PALAVRAS-CHAVE}

- Grupos Étnicos.

- Condições Sociais.

- Educação Médica.

- Especialidades Médicas.
A adoção de políticas públicas de inclusão pela Universidade Estadual de Campinas (Unicamp) desde o processo seletivo de 2005, atrelada às modificações nos vestibulares de 2014 e, mais recentemente, no de 2016, possivelmente trouxe grandes mudanças no perfil do estudante de Medicina dessa universidade. Desse modo, o objetivo do presente estudo é avaliar o perfil sociodemográfico desses acadêmicos, bem como suas pretensões na escolha da carreira médica e da futura especialidade. O estudo de corte transversal foi realizado com 290 acadêmicos do primeiro, terceiro e sexto ano da graduação médica da Unicamp por meio de um questionário anônimo aplicado após aprovação do Comitê de Ética em Pesquisa. Houve coleta de dados sociodemográficos, além dos fatores de influência para a escolha da profissão e da especialidade médica. A análise dos dados revelou uma amostra com idade média de 20-24 anos, predominantemente composta por mulheres $(63,2 \%)$, da etnia branca $(77,5 \%)$, procedentes do Estado de São Paulo (84,8\%) e de regiões interioranas (62,3\%). Para o primeiro ano, houve presença de negros $(6,6 \%)$ e diferença estatística para pardos $(22,6 \%)$, comparativa com o terceiro e sexto ano, egressos de ensino fundamental $(42,5 \%)$ e médio $(73,6 \%)$ públicos, com menor escolaridade materna (ensino médio) e renda familiar inferior $(p<0,001)$. Por sua vez, o terceiro e sexto ano eram compostos majoritariamente por alunos brancos $(76,7 \%$ e $90,3 \%$, respectivamente), oriundos de escola privada no ensino fundamental e médio, com maior escolaridade materna (ensino superior ou pós-graduação) e renda familiar mais elevada. Ainda, verificou-se variação das opções de especialidade ao longo do curso $(p<0,001)$. No primeiro ano, as especialidades mais pretendidas foram Cirurgial Ortopedia (37,7\%), Clínica Médica/Neurologia (23,6\%) e Psiquiatria (11,3\%). Para o terceiro ano, as especialidades mais desejadas foram Clínica Médica/Neurologia (40\%), Cirurgia/Ortopedia (13,4\%) e Ginecologia/Obstetrícia (13,3\%). Entre os alunos do sexto ano, as especialidades mais escolhidas foram Clínica Médica/Neurologia (24,5\%), Ginecologia/Obstetrícia (20,2\%) e Cirurgia/Ortopedia $(17 \%)$. Acadêmicos do primeiro ano também apresentaram diferentes aspirações abrangendo o local de trabalho futuro, com mais desejo de atuar somente no SUS ou em programas internacionais. Nesse contexto, os resultados apontam que a política de bonificação da Unicamp e suas alterações ao longo do tempo, em especial no vestibular de 2016, mostraram-se efetivas em democratizar o acesso à graduação médica, com maior pluralidade demográfica, social, econômica e étnica atreladas a variações na escolha da carreira e da especialidade médica ao longo das turmas analisadas. 


\section{KEY-WORDS}

- Ethnic Groups.

- Social Conditions.

- Education, Medical.

- Specialties, Medical.

Recebido em: 28/2/2018

Aceito em: 19/3/2018

\section{ABSTRACT}

Affirmative action policies adopted by University of Campinas (Unicamp) in 2005 entrance exams, added to 2014 modifications, and more recently in 2016, lead to a big profile change of University of Campinas medical students. Therefore, this paper aims to describe academics' sociodemographic profile, as well as their aspirations in choice of medical career and future specialty. The cross-sectional study included 290 undergraduate years 1, 3 and 6 (Y1, Y3 and Y6) medical students from Unicamp who answered an anonymous questionnaire applied after approval by the Institutional Review Board. Socio demographic data and factors potentially influencing decisions on medical career and specialty choices were analyzed. Data analysis showed a sample composed mostly of white (77.5\%), female (63.2\%), 20-24 years population, from the State of São Paulo (84.8\%) and interior (62.3\%). For Y1, there was statistically significant difference for 'pardo' (22.6\%) - it can be translated as brown-and black (6.6\%) - there wasn't black in Y3 and Y6 - ethnic groups, public school provenience - both elementary school (42.5\%) and high school (73.6\%) -, lower maternal schooling (high school, $p<0.001$ ) and lower family income ( $p$ <.001). Years 3 and 6 majority was composed of white academics $(76.7 \%$ and $90.3 \%$, respectively), coming from private schools (basic education and high school), with further maternal education (higher education/post-graduation) and bigger average income. The influence factors leading to choice for medical specialty modified $(p<0,001)$ among graduation years. Y1 students opted more frequently for 'surgery/orthopedics' (37.7\%), 'medical clinic/neurology' (23.6\%) and 'psychiatry' (11.3\%). To Y3, the most desired specialties were 'medical clinic/neurology' (40\%), 'surgery/orthopedics' (13.4\%) and 'obstetrician-gynecologist' (13.3\%). Amongst Y6 undergraduates, the most chosen specialties were 'medical clinic/neurology' (24.5\%), 'obstetrician-gynecologist' (20.2\%) and 'surgery/orthopedics' (17\%). Y1 academics also revealed different aspirations regarding intended future workplace, with larger desire to practice only on SUS or on international programs. In this context, results indicate that Unicamp inclusion policy and its modifications over the years, particularly in 2016 entrance exams, have been effective in broadening access to medical education, such as greater socio demographic, economic and ethnic plurality coupled with variations in reasons for choice of medical career and specialty among graduation years analyzed.

\section{INTRODUÇÃO}

De acordo com a Constituição Federal do Brasil de 1988, em seu artigo 60, "São direitos sociais a educação, a saúde, a alimentação, o trabalho, a moradia, o lazer, a segurança, a previdência social, a proteção à maternidade e à infância, a assistência aos desamparados, na forma desta Constituição" ${ }^{11}$. No entanto, observa-se na conjuntura atual que grande desigualdade perpassa a sociedade brasileira, ainda que o relatório de 2013 "Síntese de Indicadores Sociais - Uma Análise das Condições de Vida da População Brasileira" aponte queda da desigualdade social a partir dos anos 2000, mensurada por meio do índice de Gini, no qual 1 indicaria máxima desigualdade e 0 perfeita igualdade na distribuição de rendimentos, partindo do valor de 0,556 em 2004 para 0,507 em 2012 em relação ao Brasil².

No aspecto da educação, a desigualdade não fica restrita somente à renda familiar dos indivíduos, variando também de acordo com outros fatores, como a etnia. Assim, observou-se que, do total de estudantes brancos de 18-24 anos, 66,6\% frequentavam o ensino superior, ao passo que apenas $37,4 \%$ dos jovens pardos ou negros cursavam esse mesmo nível educacional em $2012^{2}$.

Nesse sentido, desde 2004 o governo federal tem adotado políticas que buscam ampliar o acesso ao ensino superior para a população com menor renda familiar, bem como para afrodescendentes, indígenas e deficientes ${ }^{3}$. Entre as estratégias, destaca-se a criação do Financiamento Estudantil (Fies) em 1999 e do Programa Universidade para Todos (ProUni) em 2004, além da implementação, em 2010, do Sistema de Seleção Unificada (SiSU) aos candidatos que realizam a prova do Exame Nacional do Ensino Médio (Enem)3. Assim, a Lei $\mathrm{n}^{\circ} 12.711 / 2012$ unificou as políticas afirmativas das universidades federais, estabelecendo aumento gradual da reserva 
de vagas de educação superior até 50\% no ano de 2016 aos alunos egressos do ensino médio em escolas públicas, além de abordar a questão étnica, com vagas destinadas a pardos, negros e índios, levando também em conta a renda familiar do estudante ${ }^{3}$.

Nesse sentido, as ações afirmativas objetivam assegurar o direito constitucional à educação, promovendo a representatividade de grupos pouco privilegiados na sociedade, além de garantir melhoria das condições de vida a toda a população, uma vez que a formação superior é condição importante para a ascensão socioeconômica no mercado de trabalho atualmente $^{3,4}$. Ainda, a comparação entre tipo de escola cursada no ensino médio pelos ingressantes na Universidade de Brasília (UnB) e o modo de acesso ao ensino superior - isto é, pelo método tradicional do vestibular ou pelo Sistema de Seleção Unificada (SiSU) - mostra o impacto da política de reserva de vagas. Para Medicina, apenas 1\% dos alunos era de escola pública no vestibular tradicional, em contraste com $30 \%$ dos ingressantes no curso pelo $\mathrm{SiSU}^{5}$.

Esse padrão de baixa representatividade de egressos de escola pública na graduação médica antes das políticas de inclusão repete-se em outros contextos, conforme relatado na Universidade Federal de Minas Gerais (UFMG) ${ }^{6}$ e na Universidade Federal do Espírito Santo (Ufes) 7 . Para a Universidade Estadual do Rio Grande do Norte (UERN), houve predomínio de acadêmicos de Medicina que cursaram o ensino médio público $(61,1 \%)$ no ano de 2013, após implementação do sistema de reserva de vagas 8 .

De modo sincrônico às estratégias adotadas pelas universidades federais e algumas instituições estaduais, a Universidade de São Paulo (USP) implementou em 2006 o Programa de Inclusão Social da USP (Inclusp), uma estratégia de bonificação na pontuação obtida nas duas fases do vestibular para candidatos egressos de ensino médio público no Brasil. Entretanto, mesmo com tal política, não houve diferença significativa no percentual de ingressantes na USP que cursaram totalmente ensino médio em escola pública entre 2001 (25,7\%) e $2010(25,6 \%)^{9}$.

Ainda nesse contexto, o Conselho Universitário da Universidade Estadual de Campinas (Consu) elaborou entre 2003-2004 o Programa de Ação Afirmativa e Inclusão Social (PAAIS), almejando a inclusão de alunos egressos de ensino médio da rede pública. A implantação do PAAIS foi embasada pelo fato de o ensino médio público correlacionar-se positivamente com desempenho acadêmico na universidade e.4. $^{9,}$ Após ser aplicado inicialmente no processo seletivo de 2005, observou-se para Medicina entrada triplicada de alunos oriundos de escola pública em comparação à média histórica de $10 \%$, com $14,5 \%$ dos alunos autodeclarados pretos e pardos ${ }^{4}$.

Durante o curso, candidatos que ingressaram usando a bonificação concedida pelo Programa de Ação Afirmativa e Inclusão Social apresentaram média de nota superior à dos demais candidatos e maior ganho de posição no rendimento em comparação aos demais ingressantes, sendo o curso de Medicina um dos que apresentou essas características de modo positivo e estatisticamente significativo ${ }^{10,11}$, além de o maior aproveitamento acadêmico atrelar-se a pertencer a famílias com menor nível socioeconômico e educacional, não ter feito curso preparatório para o vestibular, ser mulher e mais jovem $^{11}$.

De 2005 a 2013, o vestibular da Unicamp acrescia bonificação de 30 pontos na segunda fase do processo seletivo para alunos que cursaram integralmente o ensino médio em escolas públicas ou 40 pontos se cumprissem o critério anterior e fossem autodeclarados pretos, pardos ou indígenas (PPI $)^{10,11,4}$. No entanto, ao longo do tempo houve decréscimo do impacto da política no percentual de alunos egressos de escolas públicas / PPI matriculados na Unicamp ${ }^{12}$. Desse modo, nos processos seletivos de 2014 e 2015, os alunos egressos da rede pública passaram a receber 60 pontos extras na nota final ou 80 pontos se também autodeclarados $\mathrm{PPI}^{13}$.

Houve ainda nova modificação no PAAIS a partir do vestibular de 2016. Começou-se a ter adição de 60 pontos na primeira fase do processo seletivo para egressos do ensino médio público ou 80 pontos para candidatos autodeclarados pretos, pardos ou indígenas. Na segunda fase do vestibular, o acréscimo foi, respectivamente, de 90 ou 120 pontos em cada uma das provas ${ }^{14}$. Para o curso de Medicina, a modificação da pontuação no processo seletivo de 2014 elevou a porcentagem final de matriculados nesta categoria de 14,5\% (2013) para 32,4\% (2014), ao passo que a segunda mudança, ocorrida no vestibular de 2016, impactou ainda mais a quantidade final de matriculados egressos de escola pública, partindo de 15,5\% (2015) para 68,2\% (2016) ${ }^{12}$.

Assim, observa-se que a literatura acerca do impacto do Programa de Ação Afirmativa e Inclusão Social no perfil dos alunos da Unicamp aborda diversas vezes a temática em relação aos primeiros anos de implementação da política afirmativa na universidade. Entretanto, ainda não houve estudos que avaliassem as mudanças recentes, em especial após as modificações das regras de bonificação que entraram em vigor nos vestibulares de 2014 e 2016. No caso do curso médico, que apresentou 221 candidatos para cada vaga oferecida na pri- 
meira fase do processo seletivo da Universidade Estadual de Campinas em $2017^{15}$, notou-se - com base nos dados coletados pela Comissão Permanente para os Vestibulares/Comvest que essa política de ações afirmativas alterou o perfil sociodemográfico do estudante de Medicina da Unicamp ${ }^{16}$. Paralelamente, espera-se que tal modificação se estenda para além da graduação, isto é, que modifique também o perfil do profissional médico. Desse modo, é importante avaliar as pretensões destes novos alunos, de forma a ajustar possíveis descompassos da grade curricular. Ainda, é significativa a análise das aspirações destes estudantes em relação à carreira médica, a fim de verificar os efeitos que a maior pluralidade étnica e socioeconômica advinda de programas afirmativos propicia tanto na graduação médica quanto possivelmente no profissional em si. Desse modo, o objetivo do presente estudo é avaliar o perfil sociodemográfico desses acadêmicos, bem como suas pretensões na escolha da carreira médica e da futura especialidade.

\section{MATERIAIS E MÉTODOS}

Este estudo de corte transversal analisou dados relativos a fatores sociodemográficos do curso de Medicina da Faculdade de Ciências Médicas da Universidade Estadual de Campinas. Após aprovação do Comitê de Ética em Pesquisa da Faculdade de Ciências Médicas, foram convidados todos os estudantes do primeiro, terceiro e sexto ano de graduação para responder a um questionário no ano de 2016, totalizando 355 alunos, com 336 presentes no dia da aplicação. Os questionários foram aplicados pelos fiscais de sala para os 290 alunos que concordaram voluntariamente em participar da pesquisa, sendo preenchidos de forma anônima após leitura e assinatura do Termo de Consentimento Livre e Esclarecido. A taxa de resposta total foi de $86,30 \%$ ( $n=290$ em 336 presentes), sendo $95,53 \%$ do primeiro ano ( $\mathrm{n}=106$ em 111 presentes), 73,17\% do terceiro ano ( $n=90$ em 123 presentes) e $92,15 \%$ do sexto ano (n = 94 em 102 presentes).

A variável numérica avaliada foi a idade do participante, ao passo que as variáveis categóricas foram gênero, etnia, instituição em que cursou o ensino fundamental e o médio (se pública ou privada); realização de curso pré-vestibular; procedência (região/estado - Estado de São Paulo; Região Sudeste; Centro-Oeste; Nordeste; Norte; Sul -, interior/capital e porte da cidade de origem - se pequena, média, grande ou metrópole); renda familiar (até 1 salário mínimo / SM; 1-5 SM; 10-30 SM; mais de 30 SM); participação na renda; nível de escolaridade da mãe (ensino fundamental, médio, superior, pós-graduação e completo/incompleto); presença de profissional da área da saúde na família; fatores de influência para escolha da profissão médica (cuidar de pessoas; responsabilidade social; empatia e amor ao próximo; vocação e aptidão; características da profissão); área de preferência para exercício da medicina (sistema público ou privado); local de moradia após o término da graduação (cidade de origem; Campinas; centro urbano maior; centro urbano menor; outro país); fatores de influência para escolha da especialidade médica (garantia de emprego no futuro; boa remuneração; status social; conselho de amigos, parentes, professores ou profissionais da área; flexibilidade do horário de trabalho; razões pessoais; contato com paciente; influência de professores ou experiência educacional durante a graduação); local pretendido de atuação profissional (clínica privada/consultório próprio; hospital; instituições de cuidado mental; asilo; programas internacionais; não sei ainda; outros); especialidade médica pretendida (Anestesiologia; Cirurgia/ Ortopedia; Clínica Médica/Neurologia; Pediatria; Ginecologia e Obstetrícia; Psiquiatria; não sei; outros) no momento de preenchimento do questionário.

Para análise de dados, foi utilizado o programa computacional The SAS System for Windows (Statistical Analysis System), versão 9.2.SAS Institute Inc, 2002-2008, Cary, NC, USA. Para comparação das variáveis categóricas entre os anos e as opções de especialidade médica foram utilizados os testes Qui-Quadrado ou exato de Fisher (para valores esperados menores que 5). Na comparação das variáveis numéricas foi utilizado o teste de Kruskal-Wallis, devido à ausência de distribuição normal das variáveis. $\mathrm{O}$ nível de significância adotado para os testes estatísticos foi de 5\%, ou seja, p < 0,05.

\section{RESULTADOS}

A avaliação dos fatores sociodemográficos em relação ao ano do curso (Tabela 1) revelou uma amostra com idade média de 22,8 anos, predominantemente composta por mulheres $(63,2 \%)$ da etnia branca $(77,5 \%)$. No aspecto da etnia, houve diferença estatística significativa, no primeiro ano predominando $22,6 \%$ de pardos e $6,6 \%$ de negros, ao passo que o terceiro ano varia por ter $15,5 \%$ de amarelos, e no sexto ano há predominância de 90,3\% de brancos ( $\mathrm{p}<0,001)$. 


\begin{tabular}{|c|c|c|c|c|c|c|}
\hline Avalia & $\begin{array}{c}\text { TABELA } 1 \\
\text { ção dos fatores sociodemográficos em } \mathbf{r}\end{array}$ & relação ao a & no do curs & & & \\
\hline & & $\begin{array}{c}\text { Amostra } \\
\text { Total N (\%) }\end{array}$ & $\begin{array}{l}1^{\circ} \text { ano } \\
\mathrm{N}(\%)\end{array}$ & $\begin{array}{l}3^{\circ} \text { ano } \\
\mathrm{N}(\%)\end{array}$ & $\begin{array}{l}6^{\circ} \text { ano } \\
\mathrm{N}(\%)\end{array}$ & $\mathbf{P}$ \\
\hline Total de alunos por ano do curso & & $355(100)^{*}$ & $123(34,7)^{*}$ & $125(35,2)^{*}$ & $107(30,1)^{*}$ & - \\
\hline Total de respondentes por ano do curso & & $290(100)$ & $106(36,6)$ & $90(31)$ & $94(32,4)$ & - \\
\hline Idade média & & 22,8 anos & 21,3 anos & 22,3 anos & 25 anos & $<0,001$ \\
\hline Gênero & Masculino & $106(36,8)$ & $42(39,6)$ & $35(39,3)$ & $29(31,2)$ & 0,393 \\
\hline & Feminino & $182(63,2)$ & $64(60,4)$ & $54(60,7)$ & $64(68,8)$ & \\
\hline Etnia & Branco & $224(77,5)$ & $71(67)$ & $69(76,7)$ & $84(90,3)$ & $<0,001$ \\
\hline & Negro & $7(2,4)$ & $7(6,6)$ & $0(0)$ & $0(0)$ & \\
\hline & Pardo & $33(11,4)$ & $24(22,6)$ & $7(7,8)$ & $2(2,2)$ & \\
\hline & Amarelo & $25(8,7)$ & $4(3,8)$ & $14(15,5)$ & $7(7,5)$ & \\
\hline Ensino fundamental & Escola pública & $70(24,1)$ & $45(42,5)$ & $17(18,9)$ & $8(8,5)$ & $<0,001$ \\
\hline & Escola privada & $220(75,9)$ & $61(57,5)$ & $73(81,1)$ & $86(91,5)$ & \\
\hline Ensino médio & Escola pública & $116(40)$ & $78(73,6)$ & $28(31,1)$ & $10(10,6)$ & $<0,001$ \\
\hline & Escola privada & $174(60)$ & $28(26,4)$ & $62(68,9)$ & $84(89,4)$ & \\
\hline Cursinho pré-vestibular & Não & $51(17,6)$ & $15(14,1)$ & $16(17,8)$ & $20(21,3)$ & 0,417 \\
\hline & Sim & $239(82,4)$ & $91(85,9)$ & $74(82,2)$ & $74(78,7)$ & \\
\hline Procedência - regiões & Estado de São Paulo & $246(84,8)$ & $94(88,7)$ & $74(82,2)$ & $78(83)$ & 0,444 \\
\hline & Região Sudeste & $20(6,9)$ & $6(5,7)$ & $5(5,6)$ & $9(9,6)$ & \\
\hline & Região Centro-Oeste & $7(2,4)$ & $2(1,9)$ & $1(1,1)$ & $4(4,3)$ & \\
\hline & Região Nordeste & $8(2,8)$ & $2(1,9)$ & $4(4,5)$ & $2(2,1)$ & \\
\hline & Região Norte & $2(0,7)$ & $1(0,9)$ & $1(1,1)$ & $0(0)$ & \\
\hline & Região Sul & $4(1,4)$ & $0(0)$ & $2(2,2)$ & $0(0)$ & \\
\hline & Outro país & $3(1)$ & $1(0,9)$ & $3(3,3)$ & $1(1)$ & \\
\hline Procedência - interior ou capital & Capital & $107(37,7)$ & $43(41)$ & $37(43)$ & $27(29)$ & 0,106 \\
\hline & Interior & $177(62,3)$ & $62(59)$ & $49(57)$ & $66(71)$ & \\
\hline Porte cidade de origem & Pequena & $53(18,3)$ & $16(15,1)$ & $17(18,9)$ & $20(21,3)$ & 0,385 \\
\hline & Média & $73(25,2)$ & $25(23,6)$ & $19(21,1)$ & $29(30,8)$ & \\
\hline & Grande & $26(8,9)$ & $11(10,4)$ & $6(6,7)$ & $9(9,6)$ & \\
\hline & Metrópole & $138(47,6)$ & $54(50,9)$ & $48(53,3)$ & $36(38,3)$ & \\
\hline Renda familiar & Até R\$ 4.400 & $49(17)$ & $31(29,2)$ & $8(8,9)$ & $10(10,7)$ & $<0,001$ \\
\hline & $\mathrm{R} \$ 4.400-\mathrm{R} \$ 8.800$ & $76(26,3)$ & $28(26,4)$ & $31(34,4)$ & $17(18,3)$ & \\
\hline & $\mathrm{R} \$ 8.800-\mathrm{R} \$ 17.600$ & $90(31,1)$ & $25(23,6)$ & $28(31,1)$ & $37(39,8)$ & \\
\hline & $\mathrm{R} \$ 17.600-\mathrm{R} \$ 26.400$ & $43(14,9)$ & $15(14,2)$ & $11(12,2)$ & $17(18,3)$ & \\
\hline & Acima de $\mathrm{R} \$ 26.400$ & $31(10,7)$ & $7(6,6)$ & $12(13,3)$ & $12(12,9)$ & \\
\hline Participação na renda & Nenhuma & $281(96,9)$ & $101(95,3)$ & $86(9,6)$ & $94(100)$ & 0,302 \\
\hline & Trabalho e ajuda financeira da família & $6(2)$ & $3(2,9)$ & $3(3,3)$ & $0(0)$ & \\
\hline & Trabalho e ajudo na renda familiar & $1(0,3)$ & $1(0,9)$ & $0(0)$ & $0(0)$ & \\
\hline & Trabalhos ocasionais, sem remuneração fixa & $2(0,7)$ & $1(0,9)$ & $1(1,1)$ & $0(0)$ & \\
\hline Escolaridade materna & Ensino fundamental incompleto & $7(2,4)$ & $6(5,7)$ & $1(1,1)$ & $0(0)$ & $<0,001$ \\
\hline & Ensino fundamental completo & $8(2,8)$ & $3(2,8)$ & $1(1,1)$ & $4(4,3)$ & \\
\hline & Ensino médio incompleto & $8(2,8)$ & $7(6,6)$ & $0(0)$ & $1(1)$ & \\
\hline & Ensino médio completo & $36(12,4)$ & $20(18,9)$ & $8(8,9)$ & $8(8,5)$ & \\
\hline & Ensino superior incompleto & $28(9,7)$ & $12(11,3)$ & $11(12,2)$ & $5(5,3)$ & \\
\hline & Ensino superior completo & $94(32,4)$ & $25(23,6)$ & $27(30)$ & $42(44,7)$ & \\
\hline & Pós-graduação incompleta & $28(9,6)$ & $12(11,3)$ & $10(11,1)$ & $6(6,4)$ & \\
\hline & Pós-graduação completa & $81(27,9)$ & $21(19,8)$ & $32(35,6)$ & $28(29,8)$ & \\
\hline Profissional área da saúde na família & Não & $124(42,9)$ & $44(41,9)$ & $36(40)$ & $44(46,8)$ & 0,015 \\
\hline & Parente $1^{\circ}$ grau médico & $44(15,2)$ & $7(6,7)$ & $17(18,9)$ & $20(21,3)$ & \\
\hline & Parente $1^{\circ}$ grau área da saúde & $34(11,8)$ & $15(14,3)$ & $12(13,3)$ & $7(7,5)$ & \\
\hline & Parente $2^{\circ}$ grau médico & $12(4,1)$ & $4(3,8)$ & $3(3,3)$ & $5(5,3)$ & \\
\hline & Parente $2^{\circ}$ grau área da saúde & $4(1,4)$ & $2(1,9)$ & $1(1,1)$ & $1(1,1)$ & \\
\hline & Parente $3^{\circ} / 4^{\circ}$ grau médico & $37(12,8)$ & $12(11,4)$ & $12(13,4)$ & $13(13,8)$ & \\
\hline & Parente $3^{\circ} / 4^{\circ}$ grau área da saúde & $34(11,8)$ & $21(20)$ & $9(10)$ & $4(4,2)$ & \\
\hline
\end{tabular}

*Utilizado o número total de alunos nos respectivos nos anos do curso, conforme informações fornecidas pela Coordenação de Ensino em Graduação de Medicina da Unicamp. 


\begin{tabular}{|c|c|c|c|c|c|c|c|}
\hline \multicolumn{8}{|c|}{ TABELA 2} \\
\hline & & & $\begin{array}{l}1^{\circ} \text { ano } \\
\text { N (\%) }\end{array}$ & $\begin{array}{l}3^{\circ} \text { ano } \\
\text { N }(\%)\end{array}$ & $\begin{array}{l}6^{\circ} \text { ano } \\
\text { N (\%) }\end{array}$ & $\begin{array}{c}\text { Total } \\
\text { N }(\%)\end{array}$ & p \\
\hline \multirow{10}{*}{$\begin{array}{l}\text { Fatores levados em } \\
\text { consideração para } \\
\text { escolha da profissão } \\
\text { médica }\end{array}$} & Cuidar de pessoas & Sim & $66(62,3)$ & $51(56,7)$ & $37(39,4)$ & $154(53,1)$ & 0,004 \\
\hline & & Não & $40(37,7)$ & $49(43,3)$ & $57(60,6)$ & $136(46,9)$ & \\
\hline & Responsabilidade social & Sim & $24(22,6)$ & $15(16,7)$ & $7(7,5)$ & $46(15,9)$ & 0,013 \\
\hline & & Não & $82(77,4)$ & $75(83,3)$ & $87(92,5)$ & $244(84,1)$ & \\
\hline & Empatia e amor ao próximo & $\operatorname{Sim}$ & $10(9,4)$ & $13(14,4)$ & $3(3,2)$ & $26(9)$ & 0,028 \\
\hline & & Não & $96(90,6)$ & $77(85,6)$ & $91(96,8)$ & $264(91)$ & \\
\hline & Vocação e aptidão & $\operatorname{Sim}$ & $28(26,4)$ & $31(34,4)$ & $26(27,7)$ & $85(29,3)$ & 0,428 \\
\hline & & Não & $78(73,6)$ & $59(65,6)$ & $68(72,3)$ & $205(70,7)$ & \\
\hline & Características da profissão & Sim & $34(32,1)$ & $39(43,3)$ & $41(43,6)$ & $114(39,3)$ & 0,160 \\
\hline & & Não & $72(67,9)$ & $51(67,7)$ & $53(56,4)$ & $176(60,7)$ & \\
\hline \multirow{6}{*}{$\begin{array}{l}\text { Local de atuação } \\
\text { profissional após } \\
\text { o término da } \\
\text { graduação }\end{array}$} & Voltar para a cidade de origem & & $31(30,4)$ & $24(27)$ & $25(26,9)$ & $80(28,2)$ & 0,355 \\
\hline & Permanecer em Campinas & & $36(35,3)$ & $27(30,3)$ & $42(45,2)$ & $105(37)$ & \\
\hline & Morar em centro urbano menor & & $22(21,6)$ & $20(22,5)$ & $16(17,2)$ & $58(20,4)$ & \\
\hline & Morar em centro urbano maior & & $2(1,9)$ & $1(1,1)$ & $0(0)$ & $3(1,1)$ & \\
\hline & Morar em zona rural & & $6(5,9)$ & $7(7,9)$ & $7(7,5)$ & $20(7)$ & \\
\hline & Morar em outro país & & $5(4,9)$ & $10(11,2)$ & $3(3,2)$ & $18(6,3)$ & \\
\hline \multirow{4}{*}{$\begin{array}{l}\text { Pretensão na área } \\
\text { de exercício da } \\
\text { medicina }\end{array}$} & Apenas SUS & & $18(17)$ & $6(6,7)$ & $5(5,4)$ & $29(10)$ & 0,024 \\
\hline & Apenas saúde privada & & $8(7,5)$ & $6(6,7)$ & $9(9,7)$ & $23(8)$ & \\
\hline & SUS e saúde privada & & $80(75,5)$ & $75(83,3)$ & $79(84,9)$ & $234(81)$ & \\
\hline & Apenas carreira acadêmica & & $0(0)$ & $3(3,3)$ & $0(0)$ & $3(1)$ & \\
\hline \multirow{8}{*}{$\begin{array}{l}\text { Intenção de } \\
\text { localidade de } \\
\text { trabalho }\end{array}$} & Clínica/consultório próprio & & $12(11,3)$ & $19(21,1)$ & $35(38,3)$ & $67(23,1)$ & 0,001 \\
\hline & Hospital & & $52(49,1)$ & $41(45,6)$ & $41(43,6)$ & $134(46,2)$ & \\
\hline & Instituições de cuidado mental & & $6(5,7)$ & $3(3,4)$ & $0(0)$ & $9(3,1)$ & \\
\hline & Instituições governamentais & & $0(0)$ & $1(1,1)$ & $0(0)$ & $1(0,3)$ & \\
\hline & Programas internacionais & & $10(9,4)$ & $4(4,4)$ & $2(2,1)$ & $16(5,5)$ & \\
\hline & Não sei ainda & & $14(13,2)$ & $14(15,6)$ & $8(8,5)$ & $36(12,4)$ & \\
\hline & Outros & & $2(1,9)$ & $4(4,4)$ & $4(4,3)$ & $10(3,5)$ & \\
\hline & Misto de locais acima & & $10(9,4)$ & $4(4,4)$ & $3(3,2)$ & $17(5,9)$ & \\
\hline
\end{tabular}

No caso do local de realização do ensino médio, o primeiro ano teve $73,6 \%$ de alunos respondentes oriundos de escola pública, enquanto no terceiro ano houve $68,9 \%$ provenientes de escola privada e no sexto ano $89,4 \%$ de escola privada ( $\mathrm{p}<0,001)$. $\mathrm{O}$ aspecto da renda familiar apresentou diferença estatística significativa ( $\mathrm{p}<0,001)$, com predomínio de até $\mathrm{R} \$ 4.400(29,2 \%)$ no primeiro ano, $\mathrm{R} \$ 4.400-\mathrm{R} \$ 8.800$ $(34,4 \%)$ no terceiro ano, bem como $\mathrm{R} \$ 8.800-\mathrm{R} \$ 17.600(39,8 \%)$ no sexto ano. Para escolaridade materna, observou-se diferença estatística $(\mathrm{p}<0,001)$, destacando-se no primeiro ano ensino médio completo $(18,9 \%)$, no terceiro e no sexto ano ensino superior completo ( $30 \%$ e $44,7 \%$, respectivamente) e pós-graduação completa (35,6\% e $29,8 \%$, respectivamente). Em relação à presença de profissional da área da saúde na família, houve diferença significativa $(p=0,015)$, com predomínio de parente de terceiro/quarto grau da área da saúde $(20 \%)$ no primeiro ano, enquanto no terceiro/sexto ano houve maior presença de parente de primeiro grau médico, respectivamente, $18,9 \%$ e $21,3 \%$.

Os fatores de influência para a escolha da profissão médica de acordo com o ano do curso estão descritos na Tabela 2. Os aspectos mais relevantes para os alunos foram cuidar de pessoas $(\mathrm{p}=0,004)$, características da profissão $(\mathrm{p}=0,160)$, vocação e aptidão $(\mathrm{p}=0,428)$, responsabilidade social $(\mathrm{p}=0,013)$ e 
empatia/amor ao próximo ( $\mathrm{p}=0,028)$. Os fatores cuidar de pessoas, responsabilidade social e empatialamor ao próximo foram positivamente relevantes para o primeiro/terceiro ano, mas não para o sexto ano. Para o fator de pretensão na área de exercício da medicina $(\mathrm{p}=0,024)$, destacou-se no primeiro ano "apenas SUS" (17\%), ao passo que o terceiro/sexto ano optou majoritariamente por "SUS e saúde privada", respectivamente, $83,3 \%$ e $84,9 \%$. A variável intenção de localidade de trabalho mostrou o primeiro ano diferindo ao optar mais por "instituições de cuidado mental" (5,7\%) e "programas internacionais" $(9,4 \%)$, ao passo que o terceiro ano escolheu "clínica/consultório próprio" (21,1\%), do mesmo modo que o sexto ano $(38,3 \%)(p=0,001)$.

O Gráfico 1 indica os fatores ponderados durante a escolha pela especialidade médica $(\mathrm{p}<0,001)$. Os aspectos relatados pelo primeiro ano como importantes foram garantia de emprego (16,5\%) e razões pessoais $(42,7 \%)$. Para o terceiro ano, os fatores foram garantia de emprego $(17,8 \%)$, boa remuneração $(11,1 \%)$, contato com o paciente $(25,6 \%)$ e influência de professores ou experiência educacional durante a graduação (15,5\%). Por outro lado, para o sexto ano, os elementos de maior relevância foram flexibilidade no horário de trabalho
(13,2\%) e influência de professores ou experiência educacional durante a graduação $(17,6 \%)$.

A opção pela especialidade médica pretendida pelos alunos conforme o ano da graduação apresentou diferença estatística ( $p<0,001$ ), conforme apresentado no Gráfico 2. No primeiro ano, as especialidades mais pretendidas foram Cirurgia/Ortopedia (37,7\%), Clínica Médica/Neurologia $(23,6 \%)$ e Psiquiatria (11,3\%), ao passo que no terceiro ano as mais desejadas foram Clínica Médica/Neurologia (40\%), Cirurgia/Ortopedia (13,4\%) e Ginecologia/Obstetrícia $(13,33 \%)$. Entre os alunos do sexto ano, as áreas mais escolhidas foram Clínica Médica/Neurologia (24,5\%), Ginecologia/ Obstetrícia (20,2\%) e Cirurgia/Ortopedia (17\%). A comparação entre a opção pela área de especialidade médica pretendida e o local de realização do ensino médio é descrita no Gráfico 3. Houve diferença estatística $(p=0,047)$ entre as opções. A maioria dos alunos que optaram por Clínica Médica/Neurologia, Ginecologia/Obstetrícia, diagnóstico por imagem ou laboratorial, Anestesiologia e Pediatria era proveniente de escolas particulares, ao passo que os egressos de escola pública escolheram majoritariamente Cirurgia/Ortopedia, Medicina Social e Psiquiatria.

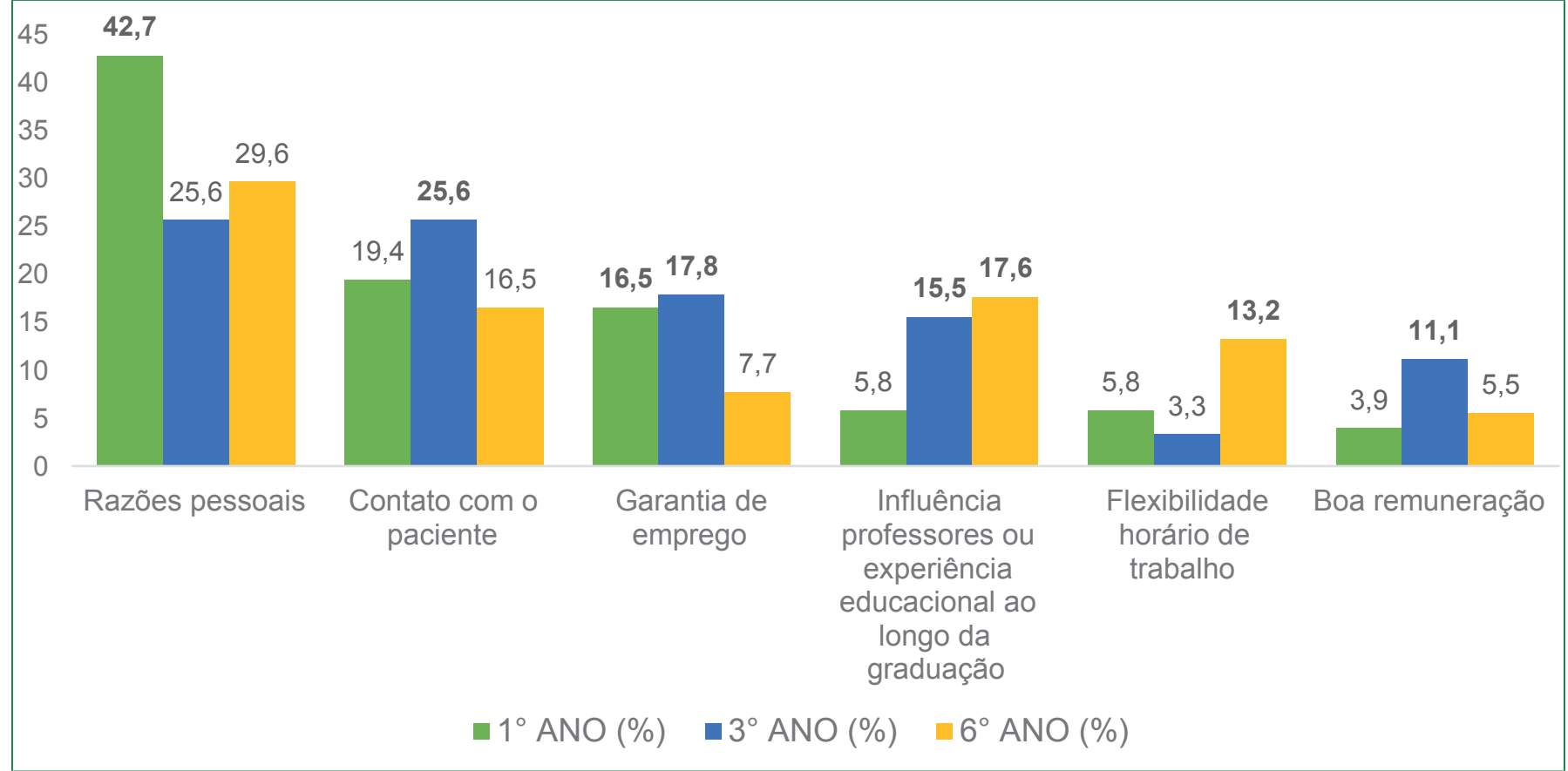




\section{GrÁfico 2}

Especialidade médica pretendida e variação entre os anos $(p<0,001)$

Clínica Médica/Neurologia

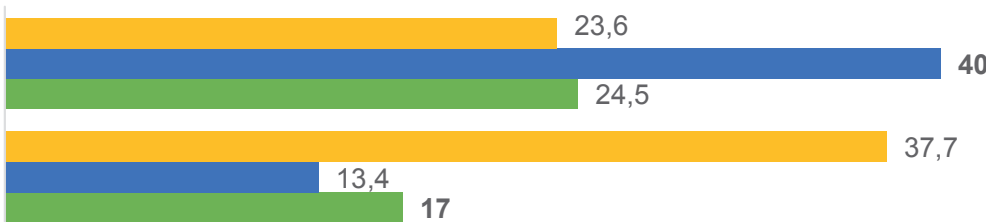

Cirurgia/Ortopedia

17

Ginecologia e Obstetrícia

\section{2,8}

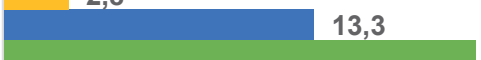

20,2

\section{Psiquiatria}

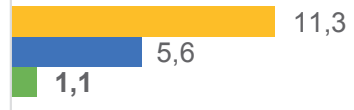

Pediatria

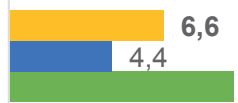

Anestesiologia

$$
1,9
$$

1,2

Não sei

Outros

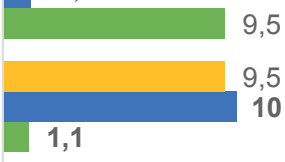

$$
1,1
$$

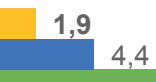

\section{9,6}

0

$5 \quad 10$

$\square 1^{\circ}$ ano (\%) $\quad \square 3^{\circ}$ ano (\%) $\quad 6^{\circ}$ ano (\% )

\section{Gráfico 3}

Opção pela especialidade médica pretendida e local de realização do ensino médio $(p=0,0047)$

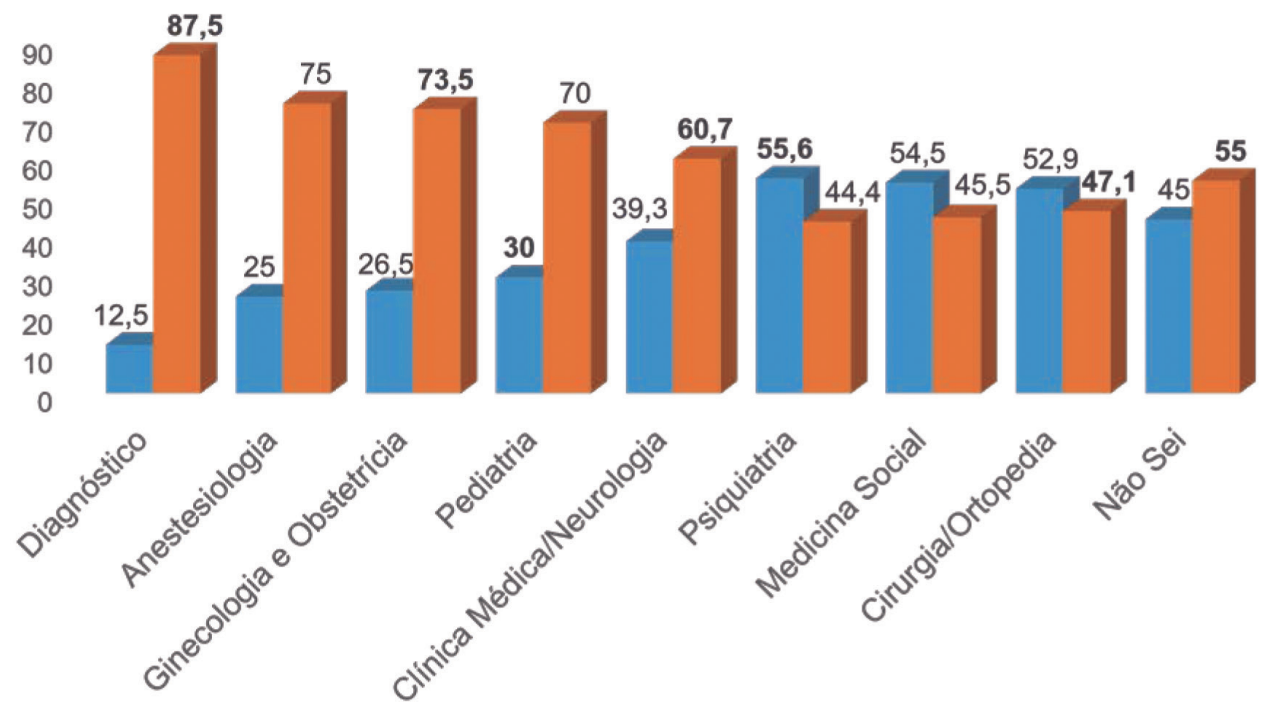

= Escola Pública (\%) Escola Privada (\%) 


\section{DISCUSSÃO}

O Brasil é um dos países com a maior desigualdade no acesso à educação, sendo a faixa de renda familiar estreitamente relacionada ao maior acesso ao ensino superior. As políticas de inclusão no ensino universitário estabelecidas nas últimas décadas têm como objetivo a democratização do acesso ao ensino superior e a inserção dos integrantes de grupos sociais sub-representados, dando igualdade de oportunidades em relação aos que são de grupos predominantes. É fato que as políticas adotadas pela Unicamp, especialmente as modificações realizadas no vestibular de 2016, tiveram grande impacto na mudança do perfil dos alunos em relação tanto à procedência de escolas públicas quanto a maior pluralidade do perfil socioeconômico e étnico.

Os resultados deste estudo retratam o perfil sociodemográfico dos estudantes de Medicina da Universidade Estadual de Campinas, bem como os fatores ponderados pelos alunos na escolha da profissão médica e da futura especialidade, comparando as variações de respostas entre os anos do curso. A presença de diferenças em relação a alguns aspectos foi particular no primeiro ano, marcado por maior proveniência de estudantes de ensino fundamental e médio de escolas públicas por meio de ações afirmativas. Assim, a despeito de diferenças metodológicas, o estudo com os alunos da graduação médica da Unicamp é passível de comparação com os universitários analisados em outros estudos.

O predomínio do gênero feminino em todas as turmas analisadas da Unicamp assemelha-se ao perfil encontrado em outros graduandos em Medicina, como na Ufes ${ }^{7}$, e em escolas médicas do exterior ${ }^{17,18,19}$. Este fenômeno, considerado feminização da medicina, tem acontecido no Brasil e em grande parte do mundo nas últimas décadas, sendo foco de extensos estudos. A redução de barreiras de acesso ao ensino superior e a afirmação das mulheres no mercado de trabalho são grandes responsáveis por esse processo de progressiva diminuição das diferenças de gênero. Entretanto, tal fenômeno relaciona-se paralelamente à diminuição de remuneração da profissão e do status social do médico ${ }^{20}$. Além disto, ainda existem desigualdades de gênero no exercício da profissão, como maior oferta de cargos permanentes e de prestígio a homens e progressão mais rápida dos médicos na carreira. Esta última se explica em parte pelo fato de muitas médicas terem menor jornada de trabalho para conciliarem as demandas profissionais e familiares ${ }^{18}$.

Em relação à etnia, a maior porcentagem de alunos pardos e negros no primeiro ano em comparação ao terceiro e sexto associa-se ao impacto das políticas de inclusão. A preponderância de acadêmicos de Medicina caucasianos é histórica e sincrônica com levantamentos de outras instituições. Entre- tanto, o percentual total de alunos autodeclarados pardos $(11,4 \%)$ da Unicamp ainda é inferior ao apresentado na Ufes $(27,5 \%)$ e UERN $(38,9 \%)^{7,8}$, as quais usam também outras formas de políticas afirmativas, como cotas raciais.

No tocante ao local de realização do ensino fundamental e médio, estudantes da Unicamp do terceiro/sexto ano referiram majoritariamente serem egressos de instituições privadas de ensino. Esse padrão assemelha-se ao encontrado nos acadêmicos da Ufes e da UFMG, destoando do perfil dos alunos da UERN, no qual há predomínio de egressos de ensino fundamental e médio da rede pública, sendo que esta usa sistema de reserva de 50\% das vagas para tal público-alvo desde $2004^{6,7,8}$. Contudo, as modificações das políticas afirmativas no processo seletivo da Unicamp no ano de 2016 contribuíram para que $68,2 \%$ dos ingressantes no curso de Medicina fossem egressos de rede pública e/ou com bonificação para pretos, pardos e indígenas (PPI). No ano de 2014, houve entrada de 32,4\% de alunos bonificados por tal política, em comparação a $11,8 \%$ dos graduandos em Medicina do sexto ano, que adentraram pelo vestibular de $2010^{12}$. O perfil dos discentes de Medicina da UnB apresentou alteração semelhante na comparação entre a quantidade de alunos da rede pública que entraram pelo vestibular tradicional (1\%) e pelo SiSU (30\%)5. No entanto, na Universidade de São Paulo, mesmo após a adoção do Programa de Inclusão Social da USP (Inclusp), não houve grandes alterações no percentual de ingressantes oriundos da rede pública de ensino médio9 .

As políticas afirmativas também impactaram o perfil socioeconômico dos alunos. De acordo com o critério de estrato socioeconômico definido pela Associação Brasileira de Empresas de Pesquisa (Abep), no curso de Medicina da Unicamp predominaram as classes B2 (rendimento entre R\$ 4.852 e R\$ 9.253) e B1 (rendimento entre $\mathrm{R} \$ 9.254$ e $\mathrm{R} \$ 20.887$ ), consideradas altas para os padrões brasileiros ${ }^{21}$. Essa característica se repetiu no terceiro e no sexto ano, representantes dos estratos B1, B2 e A. Em contraponto, no primeiro ano, cuja maioria de alunos provém de políticas afirmativas, o rendimento familiar é mais baixo, com predomínio dos estratos C1 e B2.

Ainda, sabe-se que a maior escolaridade materna é considerada fator importante na composição social e econômica familiar, estando muitas vezes relacionada a maior renda, melhor desempenho acadêmico dos filhos e melhores cuidados de saúde na família. Tangente à escolaridade materna, houve diferença estatística entre as turmas analisadas na Unicamp. No primeiro ano, destacou-se ensino médio completo ou superior incompleto, enquanto para o terceiro ano sobressaiu ensino superior incompleto/completo. No caso do sexto ano, prevaleceu o ensino superior completo. Os dados obtidos em relação ao terceiro e sexto ano são consoantes com a literatura ${ }^{8.9}$. 
Também em relação aos motivos de escolha da carreira médica houve variação significativa de acordo com o ano do curso nos estudantes da Unicamp. Os fatores mais relevantes tanto para o primeiro quanto para o terceiro ano foram $\mathrm{cui-}$ dar de pessoas, responsabilidade social e empatia/amor ao próximo. $\mathrm{O}$ fator aptidão pessoal e vocacional foi mais levantado entre os alunos do sexto ano da Unicamp, bem como características da profissão. Para os alunos da UFMG, do quinto período e do internato, os motivos mais referidos foram identificação com a profissão (vocação, realização pessoal) e altruísmo (investimento social, saúde $)^{6}$. Da mesma forma, o levantamento da Ufes ${ }^{7}$ indicou como motivos para escolha da profissão médica aptidão pessoal e vocacional, além de possibilidade de poder contribuir para a sociedade, de forma semelhante às informações obtidas no presente estudo.

Em comparação à pretensão na área de exercício da medicina, destacaram-se alunos do primeiro ano desejando atuar apenas no Sistema Único de Saúde (SUS), enquanto o terceiro e o sexto ano optaram mais frequentemente por SUS e saúde privada. Esta diferença pode ser parcialmente explicada por diferentes aspirações nos anos iniciais do estudante. Entretanto, como o primeiro ano apresenta perfil étnico e socioeconômico muito diferente daquele do terceiro e sexto ano, pode-se especular que esta aspiração esteja também relacionada à proveniência do estudante. Estudantes de menor poder econômico podem representar uma parcela maior de usuários do SUS, reconhecendo seu valor como um importante direito de cidadania ou ainda apresentarem maior comprometimento social, que levaria à busca de um campo de trabalho ligado a maior contribuição comunitária do que os serviços privados.

A opção pela especialidade médica foi mais influenciada por garantia de emprego e razões pessoais para os alunos do primeiro ano; garantia de emprego, boa remuneração, contato com paciente e influência de professores ou experiência educacional durante a graduação para os do terceiro ano; já a flexibilidade de horário de trabalho foi um fator considerado pelos alunos do sexto ano. Em relação aos estudantes da Ufes, $75,5 \%$ deles acreditam que sua atuação médica possibilitará realização pessoal e financeira ${ }^{7}$, ao passo que os alunos de $\mathrm{Me}$ dicina do Centro Universitário do Estado do Pará (Cesupa) em seu primeiro ano foram influenciados em sua escolha pela afinidade pela área, pais e renda financeira; no quarto ano, por afinidade pela área, renda financeira, influência de professores e tempo livre; e no sexto ano, os fatores mais influentes foram renda financeira, tempo livre e afinidade pela área ${ }^{22}$, não tendo sido encontrados dados mais específicos sobre as motivações para escolha da residência médica na literatura brasileira. Ainda, dados da literatura apontaram que os recém-graduados médicos do Reino Unido mantiveram tendência semelhante à da Unicamp de escolha de especialidade médica, com predomínio das opções por Clínica Geral, carreiras cirúrgicas e hospitalares $^{23}$. Ainda, conforme apontado por Gasiorowski et $a l$, estudantes poloneses do primeiro/sexto ano escolheram carreira mais voltada à atenção primária do que as demais carreiras ${ }^{18}$, diferindo dos resultados da Unicamp, talvez em virtude das formas divergentes de estruturação dos sistemas de saúde entre os países.

Nesse sentido, as especialidades médicas pretendidas sofreram variações entre os anos da graduação na Universidade Estadual de Campinas. Entre a variação das especialidades ao longo dos anos, destacaram-se no primeiro ano as especialidades cirúrgicas e Psiquiatria; no terceiro ano, Clínica Médica/Neurologia, Ginecologia/Obstetrícia; no sexto ano, foram também relevantes as opções por Anestesiologia, Pediatria e outras. Em contraponto, os alunos de Medicina do Cesupa do primeiro ano optaram por Cirurgia (20,8\%) e Cancerologia $(10,4 \%)$, enquanto o quarto ano preferiu Endocrinologia $(15,7 \%)$ e Cirurgia $(11,8 \%)$, ao passo que o sexto ano escolheu mais Oftalmologia (14\%) e Pediatria $(12,3 \%)^{22}$, com alguns pontos semelhantes aos alunos da Unicamp, como maior opção por carreiras cirúrgicas no começo do curso.

A escolha da especialidade médica é multifatorial e influenciada por fatores intrínsecos, como idade, sexo, local de nascimento, atributos pessoais e preferências, bem como por fatores extrínsecos, relacionados essencialmente ao lar e ao trabalho, como circunstâncias familiares, qualidade de vida, flexibilidade, ambiente da prática geral e remuneração.

Vanasse et al. consideram que a seleção de especialidades médicas se baseia na correspondência entre as características percebidas pelo aluno da especialidade e suas necessidades de carreira $^{24}$. Essas necessidades de carreira são determinadas pelos valores do aluno, os quais, por sua vez, são definidos por uma combinação de experiências de vida escolar pré-médica, características demográficas e personalidade. Esses valores prévios do estudante serão também moldados pelas experiências da escola médica e pelos valores e cultura da instituição em que o aluno recebe formação médica ${ }^{24}$. Entretanto, este balanço é complexo e ainda pouco claro para a maioria dos formadores das escolas médicas. Ainda há grande controvérsia a respeito de qual fator ou grupo de fatores se torna o maior determinante na decisão da carreira médica. Portanto, maior clareza deste conhecimento pode contribuir para aprimorar os cursos no sentido de prover melhores ferramentas aos estudantes para realizar esta opção.

As políticas afirmativas de inclusão social na Unicamp evoluíram desde sua implementação, com destaque para a 
alteração no vestibular de 2016, que gerou uma quantidade de matriculados final (após ocupação de todas as vagas disponíveis no edital do processo seletivo de 2016) no curso de Medicina de 68,2\% de egressos de escola pública, em comparação à taxa de 15,5\% no vestibular de $2015^{4,10,11,12,13,14,25}$. Nesse sentido, grande parte dos acadêmicos de Medicina da Unicamp oriundos de escola pública são do primeiro ano, o que pode ser um viés na análise entre a área pretendida de residência médica e o local de realização do ensino médio. No entanto, percebe-se que os alunos oriundos de escola privada tenderam a optar mais por Anestesiologia, Clínica Médica/ Neurologia, diagnóstico (por imagem ou laboratorial) e Pediatria, ao passo que estudantes que cursaram ensino médio em instituição pública preferiram Cirurgia/Ortopedia, Medicina Social e Psiquiatria. Desse modo, é preciso que haja um seguimento desses alunos ao longo do tempo, a fim de se obter uma informação mais realista sobre a procedência escolar e estrato socioeconômico do estudante e o impacto deles na especialidade médica pretendida.

É importante ressaltar que os efeitos de programas de inclusão são percebidos mais rapidamente em cursos de alta demanda, o que pode ser notado pelos resultados obtidos no presente estudo com os alunos de Medicina da Unicamp. Nessa linha, o levantamento realizado na UnB apresenta o maior impacto da diversificação do perfil étnico e socioeconômico em carreiras com maior concorrência em comparação às menos concorridas. Para a graduação médica, 3,2\% dos acadêmicos selecionados pelo processo seletivo tradicional informaram pertencer a famílias com faixa salarial abaixo de três salários mínimos, opondo-se a 45,5\% dos estudantes que conquistaram a vaga através do Enem ${ }^{5}$. Igual tendência pôde ser observada no processo seletivo geral da Unicamp do ano de 2016, no qual houve $47,6 \%$ de matriculados egressos de rede pública na universidade, sendo $68,2 \%$ os ingressantes no curso de Medicina pelo PAAIS. No ano de 2014, houve entrada de $32,4 \%$ de alunos bonificados por tal política no curso médico, em comparação a 11,8\% dos graduandos em Medicina do sexto ano, os quais adentraram pelo vestibular de $2010^{13}$. Não obstante, em maio de 2017, o Conselho Universitário da Unicamp aprovou cotas étnico-raciais, cujo modelo de implementação ainda está em elaboração, programando-se o início de tal política afirmativa a partir do vestibular de $2019^{26}$.

As alterações promovidas ao longo do tempo no processo seletivo da Universidade Estadual de Campinas - com destaque para a que entrou em vigor no vestibular de 2016 provaram-se eficientes no curso de Medicina, que teve maior democratização em seu acesso, refletida em um perfil de alunos com maior pluralidade demográfica, social, econômica e étnica. Essa maior diversidade atrelou-se a variações para a escolha da carreira médica em comparação às demais turmas analisadas. Deve-se ressalvar que o presente estudo comparou alunos em diferentes fases do curso médico. Alguns aspectos são mais estáveis ao longo do tempo, sendo menos passíveis de vieses, permitindo comparar os diversos segmentos da amostra, como gênero, etnia, perfil socioeconômico, dados demográficos no geral e os fatores levados em consideração na escolha da Medicina. Entretanto, para outras variáveis, como escolha da residência médica e local preferencial de trabalho no futuro, sabe-se que outros aspectos presentes ao longo da graduação permeiam essas escolhas, sendo uma limitação deste trabalho.

\section{CONCLUSÃO}

Os perfis étnico e sociodemográfico do graduando da Universidade Estadual de Campinas sofreram modificações em relação às turmas analisadas, indicando a efetividade das medidas afirmativas de inclusão em propiciar maior diversidade social, étnica e econômica. Os participantes da pesquisa do primeiro ano apresentaram como principais motivos para opção pela Medicina cuidar de pessoas, responsabilidade social e empatia/amor ao próximo. Em relação às aspirações de local de trabalho no futuro, apresentaram maior desejo de atuação somente no SUS e em programas internacionais, bem como diferentes opções de especialidades pretendidas. Entre essas variações de opção pela especialidade, o primeiro ano tendeu a escolher mais carreiras como Psiquiatria e Cirurgia/Ortopedia. Todavia, ainda não é possível afirmar os impactos das políticas afirmativas de inclusão no profissional médico. Assim, é relevante que esses alunos sejam acompanhados ao longo do tempo, de modo a avaliar se as diferenças no perfil dos estudantes que ingressaram a partir do vestibular de 2016 permanecerão ao longo de sua graduação e após o término dela, no sentido de uma formação profissional mais abrangente e plural, que possa contribuir para uma sociedade mais igualitária.

\section{REFERÊNCIAS}

1. BRASIL. Constituição da República Federativa do Brasil. Texto constitucional originalmente publicado no Diário Oficial da União de 5 de outubro de 1988, v. 2016, p. 496, 1988. Disponível em: <https://www2.senado.leg.br/bdsf/bitstream/handle/id/518231/CF88_Livro_EC91_2016.pdf?sequence=1>.

2. IBGE. Síntese de Indicadores Sociais - Uma Análise das Condições de Vida da População Brasileira. Estudos e Pesquisas - Informação Demográfica e Socioeconômica. Número 32, p. 266, 2013. Disponível em: <http://biblioteca.ibge. gov.br/visualizacao/livros/liv66777.pdf>. 
3. BRASIL. MINISTÉRIO DA EDUCAÇÃO. Balanço Social Sesu 2003-2014. Ministério da Educação, p. 106, 2014. Disponível em: <http://portal.mec.gov.br/sesu-secretaria-de-educacao-superior/arquivos>.

4. TESSLER, Leandro; PEDROSA, Renato. PAAIS: A Experiência de um Programa de Ação Afirmativa na Unicamp. Movimento em Debate - Adunicamp, v. 1, n. 2, p. 9, 2008.

5. LOURENÇO, Vânia Maria. Limites e Possibilidades do ENEM no Processo de Democratização do Acesso à Educação Superior Brasileira. Brasília, 2016. Dissertação (Mestrado) - Faculdade de Educação. 145 f. Universidade de Brasília. Disponível em: <http:/ / repositorio.unb.br/bitstream/10482/20129/1/2016_VâniaMariaLourenço.pdf>.

6. FERREIRA, R.a. et al. O Estudante de Medicina da Universidade Federal de Minas Gerais: Perfil e Tendências. Revista da Associação Médica Brasileira, v. 46, n. 3, p. 224-31, 2000.

7. FIOROTTI, Karoline Pedroti; ROSSONI, Renzo Roldi; MIRANDA, Angélica Espinosa. Perfil do Estudante de Medicina da Universidade Federal do Espírito Santo, 2007. Revista Brasileira de Educação Médica, v. 34, n. 3, p. 355-362, 2010.

8. CARDOSO FILHO, Francisco de Assis Brito et al. Perfil do Estudante de Medicina da Universidade do Estado do Rio Grande do Norte (UERN), 2013. Revista Brasileira de Educação Médica, v. 39, n. 1, p. 32-40, 2015.

9. MATOS, Mauricio dos Santos et al. O Impacto do Programa de Inclusão Social da Universidade de São Paulo no Acesso de Estudantes de Escola Pública ao Ensino Superior Público Gratuito. Revista Brasileira de Estudos Pedagógicos, v. 93, n. 235, p. 720-742, 2012.

10. KLEINKE, Mauricio Urbano. O Vestibular Unicamp e a Inclusão Social: Experiências e Perspectivas. I Workshop de Cursinhos Pré-Vestibulares da UNESP, n. I, p. 19, 2006.

11. PEDROSA, Renato Hyuda de Luna; DACHS, José Norberto Walter. Educational and Socioeconomic Background of Undergraduates and Academic Performance: Consequences for Affirmative Action Programs at a Brazilian Research University. Imhe, p. 1-15, 2006.

12. COMISSÃO PERMANENTE PARA OS VESTIBULARES (COMVEST). Dados sobre Inclusão Social na Unicamp. Disponível em: <https://www.comvest.unicamp.br/inclusao-paais/numeros/>. Acesso em: 5 maio 2017a.

13. SANGION, Juliana. Número de Ingressantes Oriundos de Escolas Públicas Tem Aumento de 20\%. Campinas, mar. 2014, p. 591. Disponível em: <http://www.unicamp.br/ unicamp/sites/default/files/jornal/paginas/ju_591_pagina_11_web.pdf>. Acesso em: 22 mai. 2017.

14. LAURETTI, Patrícia. Vestibular 2016 Amplia Bônus. Disponível em: <http://www.unicamp.br/unicamp/ noticias/2015/08/03/unicamp-amplia-paais-no-vestibular-2016>. Acesso em: 25 jun. 2017.

15. COMISSÃO PERMANENTE PARA OS VESTIBULARES (COMVEST). Quadro Comparativo 2016/2017 na $1^{\text {a }}$ Fase. Disponível em: <http:/ /www.comvest.unicamp.br/estatisticas-comvest/vestibulares/vestibulares-anteriores / estatisticas-do-vestibular-2017/quadro-comparativo-20162017-na-1a-fase/>. Acesso em: 5 jun. 2017b.

16. COMISSÃO PERMANENTE PARA OS VESTIBULARES (COMVEST). Perfil Socioeconômico Geral - Consulta Dinâmica. Disponível em: <http://www.comvest.unicamp. br/estatisticas-comvest/estatisticas-sociais/perfil-socioeconomico/perfil-socioeconomico-geral/formulario-de-consulta/>. Acesso em: 15 out. 2017c.

17. CLELAND, Jennifer et al. Associations Between Medical School and Career Preferences in Year 1 Medical Students in Scotland. Medical Education, v. 46, n. 5, p. 473-484, 2012.

18. GASIOROWSKI, Jakub; RUDOWICZ, Elżbieta; SAFRANOW, Krzysztof. Motivation Towards Medical Career Choice and Future Career Plans of Polish Medical Students. Advances in Health Sciences Education, v. 20, n. 3, p. 709-725, 2015.

19. HEILIGERS, Phil JM. Gender Differences in Medical Students' Motives and Career Choice. BMC Medical Education, v. 12, n. 1, p. 82, 2012.

20. SCHEFFER, Mário César; CASSENOTE, Alex Jones Flores. A Feminização da Medicina no Brasil. Revista Bioética (Impr.), v. 21, n. 2, p. 268-77, 2013.

21. ASSOCIAÇÃO BRASILEIRA DE EMPRESAS DE PESQUISA. Critério Brasil 2015 e Atualização da Distribuição de Classes para 2016. Critério de Classificação Econômica Brasil, p. 1-6, 2016. Disponível em: <http://www.abep.org/ criterio-brasil >.

22. DE SOUSA, Ivy Quirino; DA SILVA, Catarina Pereira; CALDAS, Cezar Augusto Muniz. Medical Specializations: Choices and Influences. Revista Brasileira de Educação Médica, v. 38, n. 1, p. 79-86, 2013

23. SVIRKO, Elena; GOLDACRE, Michael J; LAMBERT, Trevor. Career Choices of the United Kingdom Medical Graduates of 2005, 2008 and 2009: Questionnaire Surveys. Medical Teacher, v. 35, n. 5, p. 365-375, 2013.

24. VANASSE, Alain et al. Attractiveness of Family Medicine for Medical Students: Influence of Research and Debt. Canadian Family Physician, v. 57, n. 6, 2011.

25. COMVEST. Programa de Ação Afirmativa e Inclusão Social - PAAIS. COMVEST - Comissão Permanente para os Vestibulares, 2006. Disponível em: < http://www.comvest. unicamp.br/wp-content/uploads/2017/02/paais2006. pdf >. Acesso em: 14 fev. 2018. 
26. ALVES FILHO, Manuel. Unicamp Aprova Proposta de Adoção de Cotas Étnico-Raciais. Campinas, 2017. Jornal da Unicamp - Especial Cotas Étnico-Raciais. Disponível em: <https:/ / www.unicamp.br/unicamp/ju/especial/unicamp-aprova-proposta-de-adocao-de-cotas-etnico-raciais $>$.

\section{COLABORAÇÃO DOS AUTORES}

As autoras Maria Laura Alves de Melo Silva e Joana Fróes Bragança realizaram a concepção do estudo, elaboração do questionário, análise dos dados, elaboração e revisão do artigo. O autor Helymar da Costa Machado auxiliou na análise estatística dos dados, na elaboração e revisão do artigo. As autoras Silvia Maria Riceto Ronchim Passeri e Eliana Amaral contribuíram na concepção do estudo, elaboração e revisão do artigo. Todos leram e aprovaram a versão final do artigo, as- sim como autorizaram a submissão dele à Revista Brasileira de Educação Médica. O projeto foi orientado pela autora Joana Fróes Bragança.

\section{CONFLITO DE INTERESSES}

Não há conflitos de interesse. O trabalho recebeu apoio financeiro do Programa Institucional de Bolsas de Iniciação Científica do Conselho Nacional de Desenvolvimento Científico e Tecnológico (PIBIC/CNPq).

\section{ENDEREÇO PARA CORRESPONDÊNCIA}

Dra. Joana Fróes Bragança. Faculdade de Ciências Médicas UNICAMP. Rua Alexander Flemming, 101. Cidade Universitária Zeferino Vaz. CEP 13083-970. Campinas, SP, Brasil. Telefone:+55 19 3521-9305. Email: joanafbb@gmail.com. 